\title{
Esophageal Adenocarcinoma by AJCC v7 Stage
}

National Cancer Institute

\section{Source}

National Cancer Institute. Esophageal Adenocarcinoma by A/CC v7 Stage. NCI

Thesaurus. Code C133411.

A term that refers to the staging of esophageal adenocarcinoma according to the American Joint Committee on Cancer, 7th edition. 